\title{
Liquid Crystal Ordering and Isotropic Gelation in Solutions of 4-Base-Long DNA Oligomers
}

Tommaso P. Fraccia ${ }^{1,4}$, Gregory P. Smith ${ }^{2}$, Lucas Bethge ${ }^{3}$, Giuliano Zanchetta ${ }^{1}$, Giovanni $\mathrm{Nava}^{1}$, Sven Klussmann ${ }^{3}$, Noel A. Clark ${ }^{2}$, Tommaso Bellini ${ }^{1}$

1 - Dipartimento di Biotecnologie Mediche e Medicina Traslazionale, Università di Milano, via Fratelli Cervi 93, I-20090 Segrate (MI), Italy

2 - Department of Physics and Liquid Crystal Materials Research Center, University of Colorado, Boulder, CO, 80309-0390

3 - NOXXON Pharma AG, Max-Dohrn-Str. 8-10, 10589 Berlin, Germany

4 - Dipartimento di Scienze Umane e Promozione della Qualità della Vita, Università San Raffaele di Roma, via di Val Cannuta, 247, I-00166 Roma (RM), Italy

\section{$\underline{\text { SUPPLEMENTARY INFORMATION }}$}

\section{Aggregation model}

To gain further understanding of the phase diagram, we explored the thermal stability of both LC phases and GCCG and ATTA aggregates. The thermal melting of the LC phases can be simply measured via the total transmitted intensity through crossed polarizers: as T increases, both the LC volume fraction and birefringence of the LC phase decrease. Therefore, the vanishing of the transmitted intensity marks the total melting of the LC phase. An example of such a measurement is shown in Fig. S1A (blue line), indicating that in samples of $600 \mathrm{mg} / \mathrm{ml}$ the upper limit of LC-ISO phase coexistence is about $\mathrm{T}=43{ }^{\circ} \mathrm{C}$. In the same sample we also measured the $\mathrm{T}$ dependence of $\mathrm{I}_{\mathrm{F}}$, the fluorescent emission of ethidium bromide (EtBr), a fluorophore whose fluorescence quantum yield markedly increases upon its intercalation between paired bases of DNA. The fluorescent emission of EtBr, also shown in Fig. S1A 
(red line), does not provide a quantitatively accurate determination of the fraction $\phi_{\mathrm{P}}$ of paired bases vs. T, but it certainly offers an estimate of the $\mathrm{T}$ at which most of the bases unbind.

Data in Fig. S1A can be discussed in the frame of current models for reversible linear aggregation and LC phase formation.

Due to its running-bond-type aggregation, the standard concept of melting $\mathrm{T}$ used for duplexforming oligomers cannot be applied to GCCG and ATTA because here the system does not have the two states of single and double strand, but rather a variety of aggregate lengths. The $T$ dependence of $\mathrm{I}_{\mathrm{F}}$ can instead be interpreted by deducing $\phi_{\mathrm{P}}(\mathrm{T})$ from the aggregate length distribution $\mathrm{P}(\mathrm{m})$, which we assume to be exponential, as indicated by various theoretical modeling of living polymerization:

$$
P(m)=A \exp (-m / M)
$$

where $\mathrm{m}(\mathrm{m}=1,2,3, \ldots)$ is the number of GCCG or ATTA molecules involved in the chain, $\mathrm{M}$ is the characteristic length of the distribution, $A=e^{1 / M}-1$ is the normalization coefficient. The average chain length is $\langle M\rangle=\left(1-\mathrm{e}^{-1 / \mathrm{M}}\right)^{-1}$, so that $\langle\mathrm{M}\rangle \approx \mathrm{M}$ for large $\mathrm{M}$. Since in an aggregate of $\mathrm{m}$ molecules, $1 / \mathrm{m}$ of the bases is unpaired (i.e. the terminals), we calculate

$$
\phi_{P}=1+\left(e^{1 / M}-1\right) \log \left(1-e^{-1 / M}\right)
$$

Accordingly, $\phi_{\mathrm{P}} \approx 1$ for large $\mathrm{M}$, while $\phi_{\mathrm{P}}=0.5$ - the equivalent of the melting $\mathrm{T}$ condition - when $\mathrm{M} \approx 3$, or equivalently $\langle\mathrm{M}\rangle \approx 3.5$.

As in any aggregation process, $M$ depends on inter-monomer binding strength and on $T$. Since upon chaining the GCCGs become part of a columnar structure with local rigidity, we adopt here a model recently developed for cylinder-like monomers with adhesive bases ${ }^{1}$. We evaluate the mean GCCG and ATTA aggregation length by relying on recent models inspired by the discovery of LC phases of oligomeric $\mathrm{DNA}^{2,3}$. These models evaluate the aggregation and orientational ordering of cylinders (idealized DNA duplexed oligomer) interacting base-to-base with simpllified potentials. In particular, we will here make use of the results of Ref. 1 in which the simpler square-well potential enables describing the system with computable equations.

Thus effectively, the GCCG chain can be thought of as an aggregation of 2-base-long duplexes.

As in any aggregation process, the mean aggregation number $\mathrm{M}$ depends on inter-monomer binding strength and on T. According to the model in Ref.1, in isotropic solution M can be expressed as

$$
M=\frac{1}{2}\left(1+\sqrt{1+4 l\left(c_{D N A} / w_{G C C G}\right) e^{\Delta G / k_{B} T}}\right)
$$

where $l$ is for liters, $\mathrm{w}_{\mathrm{GCCG}}$ is the molecular weight of GCCG (so that $\mathrm{c}_{\mathrm{DNA}} / \mathrm{w}_{\mathrm{GCCG}}$ is the molar concentration of GCCG, analogously for ATTA), and $\Delta \mathrm{G}$ is the free energy gain for the aggregation of each GCCG (ATTA) molecule.

With respect to its original formulation, Eq. SE3 is here simplified in two ways.

First, we have set a parameter of the model, the bond volume $V_{b}$, so to enable the use in Eq. S3 of the values for $\Delta G$ obtained from the commonly used nearest-neighbor (NN) model for DNA thermodynamics. $\mathrm{V}_{\mathrm{b}}$ is the volume that the center of mass of a each aggregated unit can explore when part of an aggregate. Specifically, we have set $\mathrm{V}_{\mathrm{b}} \mathrm{N}_{\mathrm{A}}=1 l, \mathrm{~N}_{\mathrm{A}}$ being the Avogadro number. With this assumption Eq. S3 takes the form here presented and, in the limit of weak bonding, a conditions where only monomer and dimers are present, becomes $M \approx 1+1 l\left(c_{D N A} / w_{G C C G}\right) e^{\Delta G / k_{B} T}$. This expression 
matches the weak bond limit of $\mathrm{M}$ evaluated from the law of mass action for the equilibrium between monomers and dimers:

$$
[\text { monomer }]^{2} e^{\Delta G / k_{B} T}=1 l[\text { dimer }]
$$

where the square parenthesis indicate molar concentrations. Eq. SE2 has the standard form that is the basis for the Van't Hoff equation used to extract the nearest-neighbor NN thermodynamic data for DNA hybridization from the database of melting T. In Eq. SE4 we have explicitly inserted $1 l$, ( $l$ is for liter) necessary for dimensionality, which is normally left implicit. This asymptotic identity ensures that we can use in Eq. SE1 the values for $\Delta \mathrm{G}$ from the $\mathrm{NN}$ model.

Second, we neglect entropic terms from excluded volume effects that come into play at very high concentrations and that lead here to minor corrections only. An additional effect that we neglected because it also leads to minor correction is the effect of salt. Indeed, ionic strength controls the range of electrostatic repulsion between DNA double helices and thus their effective radius and effective volume fraction. Since we have prepared our samples with no added ions, the concentration of ions is proportional to $\mathrm{c}_{\mathrm{DNA}}$, both because of dissociated counterions and because of residual ions in the lyophilized DNA after purification - see the discussion about the osmolarity measurements below. This could result in a dependence of the effective volume fraction of the concentration. We checked this effect assuming that the ionic strength is twice the one calculated form the dissociation of the phosphates, thus ranging from 2 to $4 \mathrm{M}$ in the range of interest. We evaluated the effective volume fraction on the basis of Ref.4. The resulting concentration dependence of the melting temperature is only weakly modified with respect to the ones reported below.

\section{Stability of the GCCG and ATTA aggregates}

Fig. S1B compares $\phi_{\mathrm{P}}(\mathrm{T})$ computed with $\Delta \mathrm{G}_{\mathrm{GCCG}}$ by combining Eqs. SE2 and SE3 (dotted blue line), to the measured $\mathrm{I}_{\mathrm{F}}(\mathrm{T})$ (continuous blue line) for GCCG $\mathrm{c}_{\mathrm{DNA}}=75 \mathrm{mg} / \mathrm{ml}$, a condition in which the system is always in the ISO phase. Fig. S1B also shows the corresponding computed M(T) (red dotted line).

To compare the different shapes and intensity drops of $\mathrm{I}_{\mathrm{F}}(\mathrm{T})$ obtained in the various samples, we normalized them by subtracting $\mathrm{I}_{\min }$, the value at $\mathrm{T}=90^{\circ} \mathrm{C}$, and dividing by $\mathrm{I}_{\mathrm{MAX}}$, the value at $\mathrm{T}=0{ }^{\circ} \mathrm{C}$ (with the exception of the GCCG $600 \mathrm{mg} / \mathrm{ml}$ and ATTA $400 \mathrm{mg} / \mathrm{ml}$ curves which have maximum at $\mathrm{T} \neq$ $\left.0^{\circ} \mathrm{C}\right)$. Figs. S1C and S1E thus shows $\mathrm{I}_{\mathrm{F}}{ }^{\prime}(\mathrm{T})=\left(\mathrm{I}_{\mathrm{F}}(\mathrm{T})-\mathrm{I}_{\min }\right) / \mathrm{I}_{\mathrm{MAX}}$ for the GCCG and ATTA systems, respectively. This normalization assumes that at $90^{\circ} \mathrm{C}$ most of the strands are unpaired, and enables to better account for the fact that some of the $\mathrm{I}_{\mathrm{F}}(\mathrm{T})$ curves don't show a saturating behavior even at $0^{\circ} \mathrm{C}$.

From these curves we extracted the temperature $\mathrm{T}_{\mathrm{M}}{ }^{*}$, the equivalent of the melting temperature for this running-bond type chains, so that $\mathrm{I}_{\mathrm{F}}{ }^{\prime}\left(\mathrm{T}=\mathrm{T}_{\mathrm{M}}{ }^{*}\right)=0.46$ which is half of the highest intensity drop observed, and reported them for GCCG and ATTA in Figs. S1D and S1F respectively (dots).

In Fig. S1D and S1F we also show $\mathrm{T}_{\mathrm{M}}{ }^{*}$ fits from Eqs. SE2 and SE3 with $\Delta \mathrm{G}_{\mathrm{GCCG}}$ and $\Delta \mathrm{G}_{\mathrm{ATTA}}$ as free parameter. This fit allow to determine the values of free energy and of its enthalpy and entropic parts, which are reported in table $\mathrm{S} 1$. 


\begin{tabular}{|l|c|c|c|}
\hline & $\begin{array}{c}\Delta \mathbf{H} \\
\mathbf{k c a l} / \mathbf{m o l}\end{array}$ & $\begin{array}{c}\Delta \mathbf{S} \\
\mathbf{c a l} /(\mathbf{m o l ~ K})\end{array}$ & $\begin{array}{c}\Delta \mathbf{G}(\mathbf{T}=\mathbf{3 7} \\
\mathbf{k c a l} / \mathbf{m o l}\end{array}$ \\
\hline$\Delta \mathbf{G}_{G C C G}$ & -24.6 & -66.7 & -3.91 \\
\hline$\Delta \mathbf{G}_{A T T A}$ & -14 & -37.4 & -2.4 \\
\hline
\end{tabular}

Table S1. Values of free energy obtained from $T_{M}{ }^{*}$ fits with equations SE2 and SE3. 

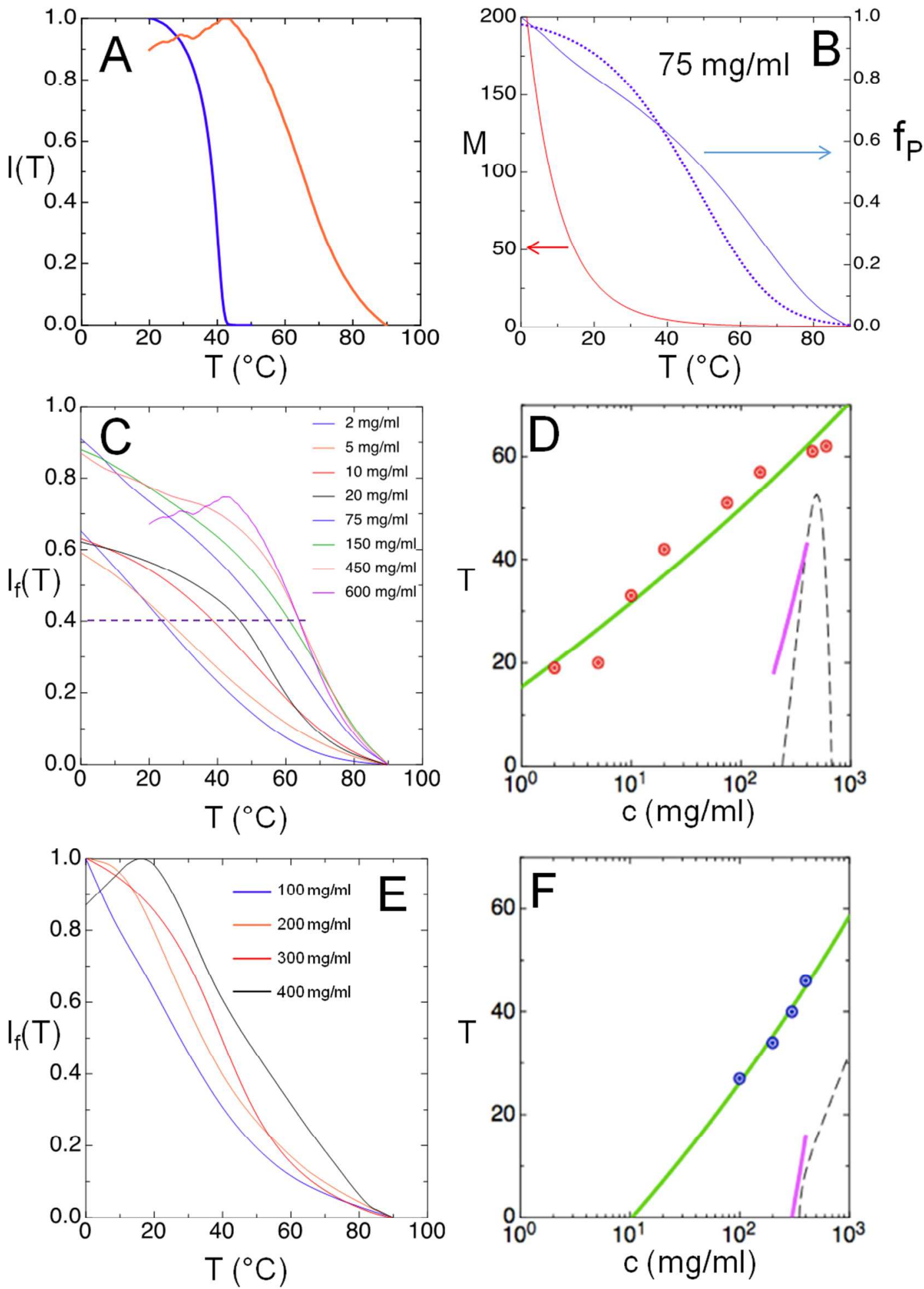

Figure S1. Thermal stability of DNA 4mers aggregates and of LC phases. Data and model predictions. 


\section{Evaluation of bonding energy contributions}

The enthalpy and entropy variations, $\Delta \mathrm{S}$ and $\Delta \mathrm{H}$, involved in the formation of the GCCG and ATTA aggregates can be assessed in the frame of the NN model by adding the various free energy contributions involved in the bonds.

To determine the value of the thermodynamic parameters of the binding of GCCG and ATTA, we adopted the following sources:

- quadruplets, i.e. pairs of adjacent Watson-Crick bonds between two distinct strands, are taken from Ref. 5. The quadruplets involved in these evaluations are GC/CG, CG/GC, AT/TA, TA/AT.

- coaxial stacking, i.e stacking interactions of chemically disconnected but physically contacting bases are taken from Ref. 6 and Ref. 7. The coaxial stacking here involved are G-G/CC and A$\mathrm{A} / \mathrm{TT}$, where the symbol - marks the position of the nick.

- dangling ends, i.e. the the stacking of an unpaired bases adjacent to a Watson-Crick pair, are taken from Ref. 5. Dangling ends involved in this study are CC/G and TT/A.

While quadruplets and dangling ends $\mathrm{NN}$ values have been widely studied and confirmed in literature, the coaxial stacking parameters are subjected to higher variations and their values highly depends on the experimental system from which they are extracted.

Therefore we adopted the choice to extract our own coaxial stacking values by subtracting all the other contributions from the experimental values obtained from our data.

On the basis of the running-bond-type aggregation the main interaction is the one between the terminal parts of two linear aggregates formed by more than 3 strands, as sketched in Fig. 1C. Therefore the $\Delta \mathrm{G}$ variation is the one calculated between the $\Delta \mathrm{G}_{0}$ of the initial state, which have two unpaired dangling ends, to the $\Delta \mathrm{G}_{1}$ of the final state, where the two dangling end pair forming a quadruplets and two coaxial stacking.

Therefore $\Delta \mathrm{G}$ is obtained from Eqz. SE4:

$$
\Delta G=\Delta G_{1}-\Delta G_{0}=\Delta G_{I}+\Delta G_{S}+\Delta G_{Q}-2 \cdot \Delta G_{D E}+2 \cdot \Delta G_{C}=\Delta G^{*}+2 \cdot \Delta G_{C}
$$

where the contributions from initiation, $\Delta G_{I}$, symmetry, $\Delta G_{S}$, quadruplets, $\Delta G_{Q}$, and dangling-ends term $\Delta G_{D E}$ are calculated from Ref. 5, and summed together gives $\Delta \mathrm{G}^{*}$, while the coaxial stacking contribution, $\Delta G_{C}$, is obtained by difference.

Fig. S2A shows the experimental $\Delta \mathrm{G}_{\mathrm{GCCG}}$ and $\Delta \mathrm{G}_{\mathrm{ATTA}}$ obtained from $\mathrm{T}_{\mathrm{M}}{ }^{*}$ fit (blue and red straight lines) and the contribution calculated from NN parameters $\Delta \mathrm{G}_{\mathrm{GCCG}}{ }_{\mathrm{GC}}$ and $\Delta \mathrm{G}^{*}{ }_{\mathrm{ATTA}}$ (blue and red dashed lines). In Fig. S2B the obtained $\Delta G_{C}$ for G-G/CC and A-A/TT coaxial stacking are reported (blue and red straight lines) and compared with those obtained from Ref. 6 (dotted lines) and Ref. 7 (dashed lines). 



Fig S2. Free-Energy contributions in GCCG and ATTA running-bond-type aggregation. (A) Experimental $\Delta G$ extracted from $T_{M}{ }^{*}$ fit (straight lines) and calculated $\Delta G^{*}$ contribution. (B) Experimental coaxial stacking energy, $\Delta G C=\left(\Delta G-\Delta G^{*}\right) / 2$, for $A-A / T T$ and $G-G / C C$ nicks (straight lines) compared with those existing in literature.

\section{Predictions for the Isotropic-LC transition}

The model used above to connect the EtBr data with the free energy of interaction among GCCGs and ATTAs can be also used to obtain an estimate of the location of the ISO-LC transition line in the phase diagram. Indeed, the model in Ref.1 predicts such transition line for volume fractions in the range $0.2-0.4$, and for aggregating cylinders of diameter $\mathrm{D}$ and axial ratio (length/D) in the range 1-3. Namely, the model indicates the aggregate length $\mathrm{L}_{\mathrm{A}}$ at which the system develops nematic order for a given volume fraction $\phi$. Interestingly, this prediction (Fig. 9 of Ref.1) does not depend much on the axial ratio in the explored range [unpublished data], and thus it is reasonable to extend it in the case of interest here.

From the model we obtain that the isotropic side of the isotropic-nematic phase coexistence is found when

$$
L_{A} \approx 0.75 D / \phi^{1.76}
$$

which, once expressed in terms of mean aggregation number M vs. DNA concentration becomes

$$
M \approx 2.06 /\left(c_{D N A} m l / g\right)^{1.76}
$$

The combination of Eq. SE7 with Eq. SE3 enables determining the T at which M satisfy Eq. SE7, and thus at which the LC phase melts.

It is useful to point out that the model includes an evaluation of the free energies of the isotropic and of the nematic phase, but not of the columnar phase. Thus, the phase boundary that it enables determining is necessarily the one between the isotropic and the nematic phase even in conditions in which the columnar ordering would be preferred over the nematic phase. For this reason, the predicted ISO-N phase can be interpreted as a ISO-LC phase. 
In Fig. S1D we plot the region of the phase diagram where LC phase are found (dashed black line, same as in Fig. $1 \mathrm{G}$ of the main text), and the prediction for $\mathrm{T}\left(\mathrm{c}_{\mathrm{DNA}}\right)$ computed with $\Delta \mathrm{G}_{\mathrm{GCCG}}$ (pink line, same as in Fig. 1G of the main text). Analogously, in Fig. S1F (and in Fig. 1F of the main text) we show $\mathrm{T}\left(\mathrm{c}_{\mathrm{DNA}}\right)$ computed with $\Delta \mathrm{G}_{A T T A}^{*}$ (green lines). Quite clearly, predictions nicely match the observed phase boundary.

\section{Osmolarity measurements}

Osmolarity was measured with a Gonotec Osmomat 030 freezing point osmometer.

Three samples were considered: (1) a homogenous GCCG solution in the ISO phase and (2) a sample prepared at $400 \mathrm{mg} / \mathrm{ml}$, showing coexistence at $\mathrm{T}=25^{\circ} \mathrm{C}$. Sample 2 was put in a capillary, centrifuged at $\mathrm{T}=25^{\circ} \mathrm{C}$ and cut at the meniscus so to separate the two phases. The two parts of the capillary were then separately diluted, generating sample $2 \mathrm{a}$ and sample $2 \mathrm{~b}$. The osmolarity of the three samples is reported in Table S2.

\begin{tabular}{|c|c|c|c|c|}
\hline Sample & $\begin{array}{c}\text { Measured } \\
\text { Osmolarity } \\
\text { O }_{\mathrm{T}}(\mathrm{mOsm} / \mathrm{l})\end{array}$ & $\begin{array}{c}\text { Expected } \\
\text { Osmolarity } \\
\mathbf{O}_{\mathrm{E}}(\mathrm{mOsm} / \mathrm{l})\end{array}$ & $\mathbf{O}_{\mathrm{T} /} \mathbf{O}_{\mathrm{E}}$ & $\mathrm{O}_{\mathrm{C}} /[\mathrm{GCCG}]$ \\
\hline 1. homogeneous ISO & 95 & 68 & 1.40 & 2.0 \\
\hline 2a. $\mathrm{N}^{*}$ (coex. with ISO) & 49 & 34 & 1.44 & 2.2 \\
\hline 2b. ISO (coex. with N*) & 77 & 58 & 1.32 & 1.6 \\
\hline
\end{tabular}

Table S2. Osmolarity of selected samples

The molar concentration of [GCCG] is measured in each sample by UV absorbance at $\lambda=260$ $\mathrm{nm}$. The osmolarity expected from [GCCG] is computed as $\mathrm{O}_{\mathrm{E}}=5 \cdot[\mathrm{GCCG}]$, assuming that all 4 counterions are dissociated. In the last column the excess osmolarity $\mathrm{O}_{\mathrm{C}}=\mathrm{O}_{\mathrm{T}}-\mathrm{O}_{\mathrm{E}}$ due to an additional contaminant is then compared with [GCCG].

Since at the original concentration at which the sample was prepared $[\mathrm{GCCG}] \approx 0.32 \mathrm{M}$, the difference in the osmolarity measured in the two coexisting phases correspond to a difference of $\mathrm{O}_{\mathrm{C}} \approx 0.2$ Osm, which could correspond to $0.1 \mathrm{M}$ of monovalent ionic contaminant or to $0.2 \mathrm{M}$ of non-ionic contaminant. For comparison, such resulting unbalance of this third component of the GCCG solution is much larger than the difference in [GCCG] between the two coexisting phases, which is of the order of $0.04 \mathrm{M}$.

\section{Equilibrium competition between G-quartets-based and pairing-based structures}

The formation of G-quartets can be described through an equilibrium condition between monomeric guanosines and guanosins associated in quartets. According to Ref. ${ }^{8}$, such equilibrium can be written as:

$$
[G]^{4}[M] K_{4}=[G 4]
$$


where $[\mathrm{G}],[\mathrm{M}]$ and $[\mathrm{G} 4]$ are the molar concentration of the monomeric guanosines, of the monovalent counterions and of the G-quartets, respectively. $\mathrm{K}_{4}$ is the binding coefficient governing the equilibrium. In our case, as in the one of Ref. ${ }^{8}$, since no salt was added to the solutions, we assume $[\mathrm{M}] \approx[\mathrm{G}]$.

Since G-quartets form a rather flat structure, the guanines being coplanar, their association in octets in which two G-quartets are stacked, is energetically favorable. The octet equilibrium can be expressed as ${ }^{8}$ :

$$
[G 4]^{2} K_{8}=[G 8][M]
$$

where $\mathrm{K}_{8}$ is the binding coefficient and [G8] is the molar concentration of the guanosine octets. Since the binding coefficients $\mathrm{K}_{4}$ and $\mathrm{K}_{8}$ have been determined ${ }^{8}$, the tendency of GCCG toward the formation of guanosine quadruplets and octets as opposed to double helical aggregates can be explicitly computed as a competitive equilibrium. The rational for this competition is that while the formation of double helical chains involves Watson-Crick bonding of all Gs and all Cs thus yielding a lower free energy per molecule, the formation of octets depends dramatically on the concentration and is thus favored at large $\mathrm{C}_{\text {DNA. }}$.

We have explicitly performed such comparison through the following steps:

1. GCCG could form octets by associating the terminal Gs of distinct molecules as in the sketch of Fig. $2 \mathrm{~L}$ of the main text. Therefore in our system $[\mathrm{G}]=2$ [GCCG].

2. while Cs don't form bonds, they certainly contribute with some stacking energy to the G-quartets, which we assume to be of the order of $\Delta \mathrm{G}_{\mathrm{S}}$, the one for "dangling ends" in the nearest-neighbor model for duplex DNA. Since this term is not present in the association of monomeric guanosines of Ref. ${ }^{8}$ we take it by modifying $\mathrm{K}_{4}$ into $\mathrm{K}_{4}{ }^{*}=\mathrm{K}_{4} \exp \left(4 \Delta \mathrm{G}_{\mathrm{S}} / \mathrm{RT}\right)$. Although this correction is not numerically very significant we took it into account because it was taken into account also in the evaluation of $\Delta \mathrm{G}_{1}$ and $\Delta \mathrm{G}_{2}$ of GCCG chaining in the main text.

3. the association of GCCG into chains is here evaluated as a duplex formation of the overhangs

$$
[G C]^{2} K_{2}=[G C 2]
$$

$\mathrm{K}_{2}=\exp (\Delta \mathrm{G} / \mathrm{RT})$ where $\Delta \mathrm{G}$ is the bond free energy discussed in the text, and should thus be $\Delta \mathrm{G}_{1}$ $>\Delta \mathrm{G}>\Delta \mathrm{G}_{2}$. [GC] is the molar concentration of half GCCG molecule, since only two bases are involved at each time in the chain bonds. Thus $[\mathrm{GC}]=2$ [GCCG]. This description is obviously a simplification over the aggregation mode of GCCG described in the main text. However, we argue that this simpler description can anyway enable us to evaluate whether, and for what bond energies and concentrations, the formation of octets can become the lowest energy state of the system.

The competitive equilibrium of the two association modes can be evaluated by expressing the total amount of molecules via the combination of Eq. S10-S13. Namely, since $[G]=[G C]$, the total amount of GCCG molecules in solution as $[\mathrm{GCCG}]_{\mathrm{T}}$ can be written as:

$$
2[G C C G]_{T}=[G]+2[G C 2]+4[G 4]+8[G 8]=[G]+2[G C]^{2} K_{2}+4[G]^{5} K_{4}+8[G]^{9} K_{4}
$$

We solved this equation determining for the two quantities

$$
\begin{gathered}
P_{2}=[G C 2] /[G C C G]_{T} \\
P_{4}=(2[G 4]+4[G 8]) /[G C C G]_{T}
\end{gathered}
$$


which correspond to the fraction of GCCG molecules involved in forming linear double helical structures and the fraction involved instead in the formation of G-quadruplexes.

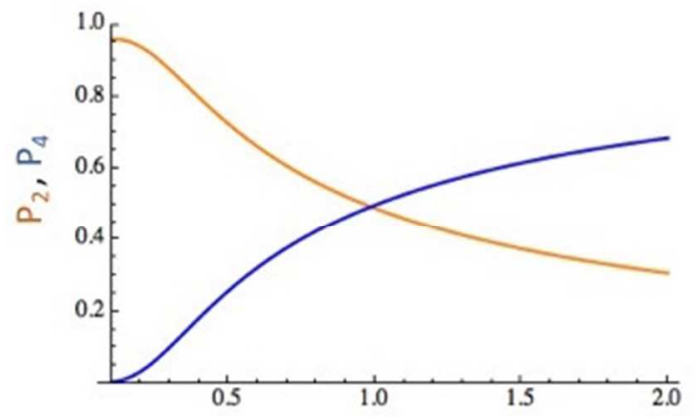

[G] (M)

Figure S3. Calculated relative concentrations of duplexes (yellow line) and G-quartets (blue line).

Parameter in the calculation ${ }^{5,8}$ :

$$
\begin{gathered}
K_{4}=2.310^{6} M^{-4} \\
K_{8}=3.310^{2} \\
\exp \left(\Delta G_{S} / R T\right) \approx 0.5
\end{gathered}
$$

Since $\mathrm{P}_{2}$ and $\mathrm{P}_{4}$ delicately depend on $\mathrm{K}_{2}$, we preferred to reverse the problem and determine the value of $\mathrm{K}_{2}$ such that $\mathrm{P}_{2}=\mathrm{P}_{4}$ at $[\mathrm{GCCG}]=0.5 \mathrm{M}$ (i.e. $[\mathrm{G}]=1 \mathrm{M}, \mathrm{c}_{\mathrm{DNA}} \approx 650 \mathrm{mg} / \mathrm{ml}$ ), which correspond to the concentration at which transition from COLX to ISO2 is observed.

We obtain $\mathrm{K}_{2} \approx 3.510^{3} \mathrm{M}^{-1}$, corresponding to $\Delta \mathrm{G} \approx-4.8 \mathrm{kcal} / \mathrm{mol}$, a value intermediate between $\Delta \mathrm{G}_{1} \approx$ $5.8 \mathrm{kcal} / \mathrm{mol}$ and $\Delta \mathrm{G}_{2} \approx-3.8 \mathrm{kcal} / \mathrm{mol}$. In Fig. $\mathrm{S} 3$ we show the dependence of $\mathrm{P}_{2}$ and $\mathrm{P}_{4}$ on [G] computed with such a choice for $\mathrm{K}_{2}$ and the parameters listed as above.

\section{$N^{*}$ phase of GCCG: chirality and cholesteric pitch}

In the main text we discussed the effects of the intercalation of EtBr into GCCG COLX phases. We find that the presence of EtBr modifies the $\mathrm{N}^{*}$ phase too. This is shown in Fig. S4. Panes S4A and S4B describe typical chiral textures of the $\mathrm{N}^{*}$ phase of neat GCCG. In the PTOM image in S4A fingerprint textures are recognized, corresponding to the helical arrangement of the $\mathrm{N}^{*}$ optical axis, as drawn in the overlaid scheme. The intensity profile along the red line is shown in Pane S4B, from which the pitch length is immediately extracted.

Addition of EtBr does not change the handedness of the $\mathrm{N}^{*}$ chirality, but induces a significant shortening of the pitch, which becomes short enough to enters the range of visible wavelengths. This is shown in Fig. S4C, where a greenish selective reflection can be noticed. Spectral analysis of the transmitted light enables determine the exact value of $\mathrm{p}$.

In both conditions (longer pitch and selective reflection), the handedness of the $\mathrm{N}^{*}$ phase can be determined by rotating the analyzer. Figs. S4D-F show PTOM images of GCCG N* region observed with orthogonal polarizers E, and rotating the analyzer counter-clockwise, D, and clockwise, F.

The intercalation of EtBr between bases ${ }^{9}$ effectively lengthens the DNA helical pitch. Given the EtBr concentration in the experiment here reported, about 1 EtBr molecule every 4 paired bases, we estimate such lengthening in about $20 \%$. This means that the angle formed by the phosphate chain with 
respect to the plane of paired bases grows from $\psi \approx 30^{\circ}$, in the case of ideal B-DNA helix, to $\psi \approx 37^{\circ}$. Starting form the pioneering contribution of Straley ${ }^{10}$, many studies have focused on the effect of chiral steric hindrance on the resulting torque. This complex problem has recently found an explicit solution by using an approach developed by Ferrarini and collaborators, treating coarse-grained DNA helical particles interacting only through excluded volume ${ }^{11}$. These studies (i) confirm previous results indicating that the steric torque expected by the B-DNA structure is right-handed, and (ii) find that h chiral torque decreases when the inclination angle $\psi$ approaches $40^{\circ}$. This result is in line with the euristic argument that the closest distance of two screw-like structures with $\psi \approx 45^{\circ}$ is attained when the angle between their axes is $90^{\circ}$, a condition in between right-handed and left-handed twist (see for example Fig. 2 of Ref. ${ }^{12}$ ). $\psi \approx 45^{\circ}$ is thus expected to be a threshold condition with zero torque. On this basis, we expect that upon inserting EtBr molecules in the chain, the steric right-handed contribution to the total torque decreases, thus favoring a stronger left-handed twist, as observed.

More complex is the understanding of the electrostatic torque favoring left-handed twist, here the dominating term. The sign and amplitude of the electrostatic torque is in fact found to depend on the details of the charge distribution ${ }^{13}$. It is thus difficult to determine what should be the expected dependence of the electrostatic torque on the inclination angle.

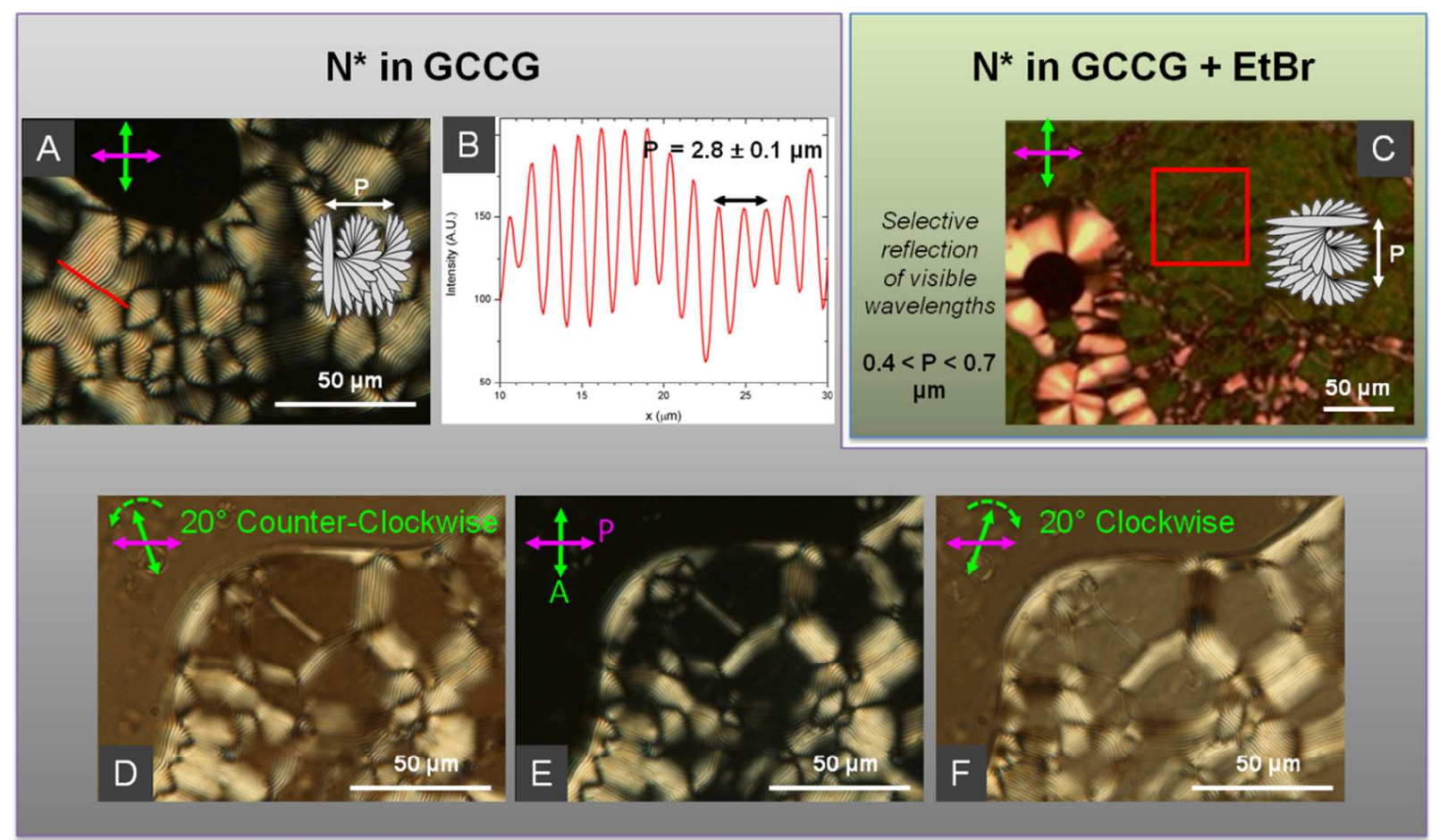

Fig. S4 - Chirality and cholesteric pitch observation in GCCG.

\section{Further observations on the ISO2 phase}

One interesting and intriguing results in this paper is the existence of an ISO2 phase. As stated in the main text, despite extensive observations, we never observed phase coexistence between ISO2 and ISO. Here we report the behavior of a specific thin $(\sim 10 \mu \mathrm{m})$ flat cell prepared at $\mathrm{c}_{\mathrm{DNA}}=770 \mathrm{mg} / \mathrm{ml}$ (and thus in ISO2), as obtained through microscope observations. Figs. S5 and S6 show two sequence of pictures pictures of such cell taken upon heating and cooling. The sample always remains isotropic (black 
through cross polarizers). The pictures enable appreciating the transition of ISO2 from gel to liquid, and the absence of any liquid-liquid interface.

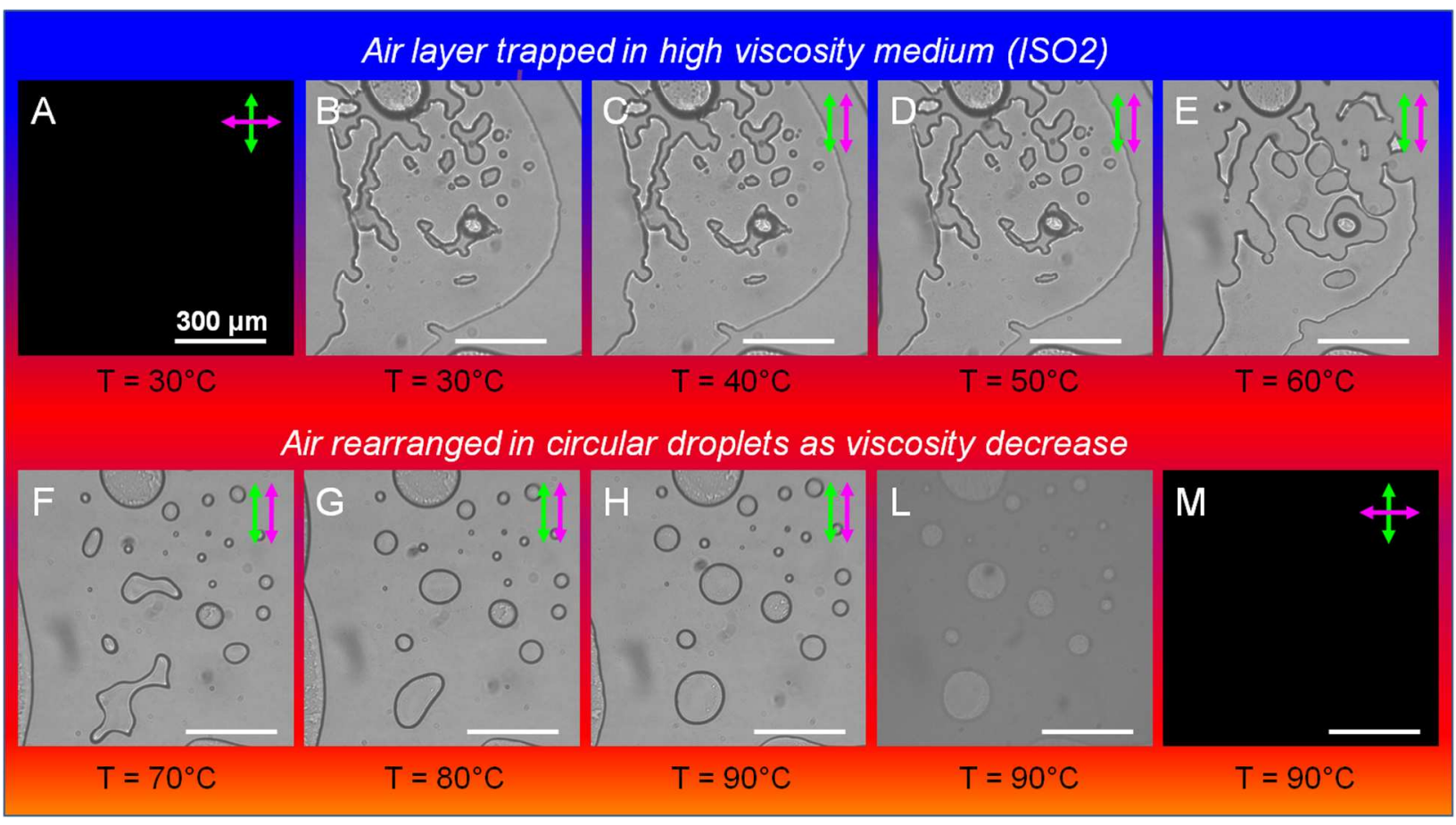

Fig. S5 - PTOM images of a GCCG ISO2 region at increasing temperatures. Pictures A and M are taken through crossed polarizers.

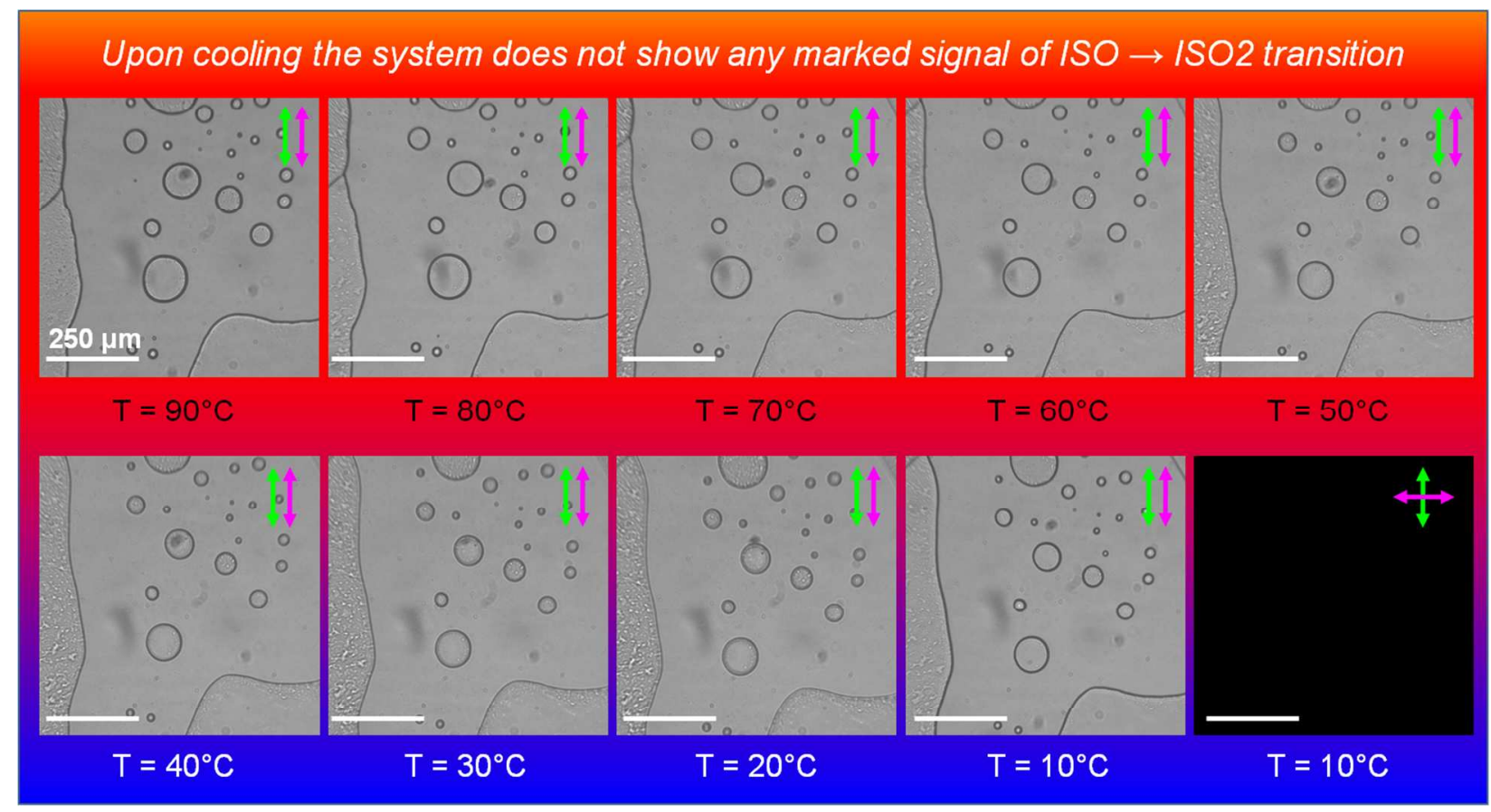

Fig. S6 - PTOM images of a GCCG ISO2 region at decreasing temperature from $90^{\circ} \mathrm{C}-10^{\circ} \mathrm{C}$. 
Fig. S5 shows a cell in which a layer of air remained trapped between one glass surface and the DNA solution, marked by the irregular boundaries in pane B. As T rises above $50{ }^{\circ} \mathrm{C}$, the ISO2 fluidity decreases, and air gathers in droplets with smooth boundaries. Pane L shows a picture taken in reflection mode, demonstrating that the bubbles are indeed air bubbles and not fluid drops: since refractive index of air is $\mathrm{n}_{\mathrm{AIR}} \sim 1$, while the refractive index of the NA solution is $\mathrm{n}_{\mathrm{SOL}} \sim 1.436$, light is reflected more from air bubbles than from the rest of the cell.

Fig. S6 shows the same cell as T is lowered back. No sign of interfaces is found, despite the fact that in all other coexistences in this system, the interfaces between coexisting phases could be observed. Indeed, given the large contribute to refractive index from DNA, even small differences in $\mathrm{c}_{\mathrm{DNA}}$ give a detectable optical effect.

\section{Further observations on the GCCG phase diagram}

Fig. S7 shows a thin flat cell of GCCG which was only partially sealed. This gave rise to evaporation from the right-hand side of the picture, and thus to a large gradient of DNA concentration, with $\mathrm{c}_{\mathrm{DNA}}$ growing from left to right. This cell enables observing at once all the phases in the phase diagram, ranging form ISO to ISO2. At the right-hand side, the GCCG solution terminates with an interface with air, that we marked in the figure by a dashed blue line. A short time before the picture was taken, the region in the figure where ISO2 is present was filled by COLX phase.

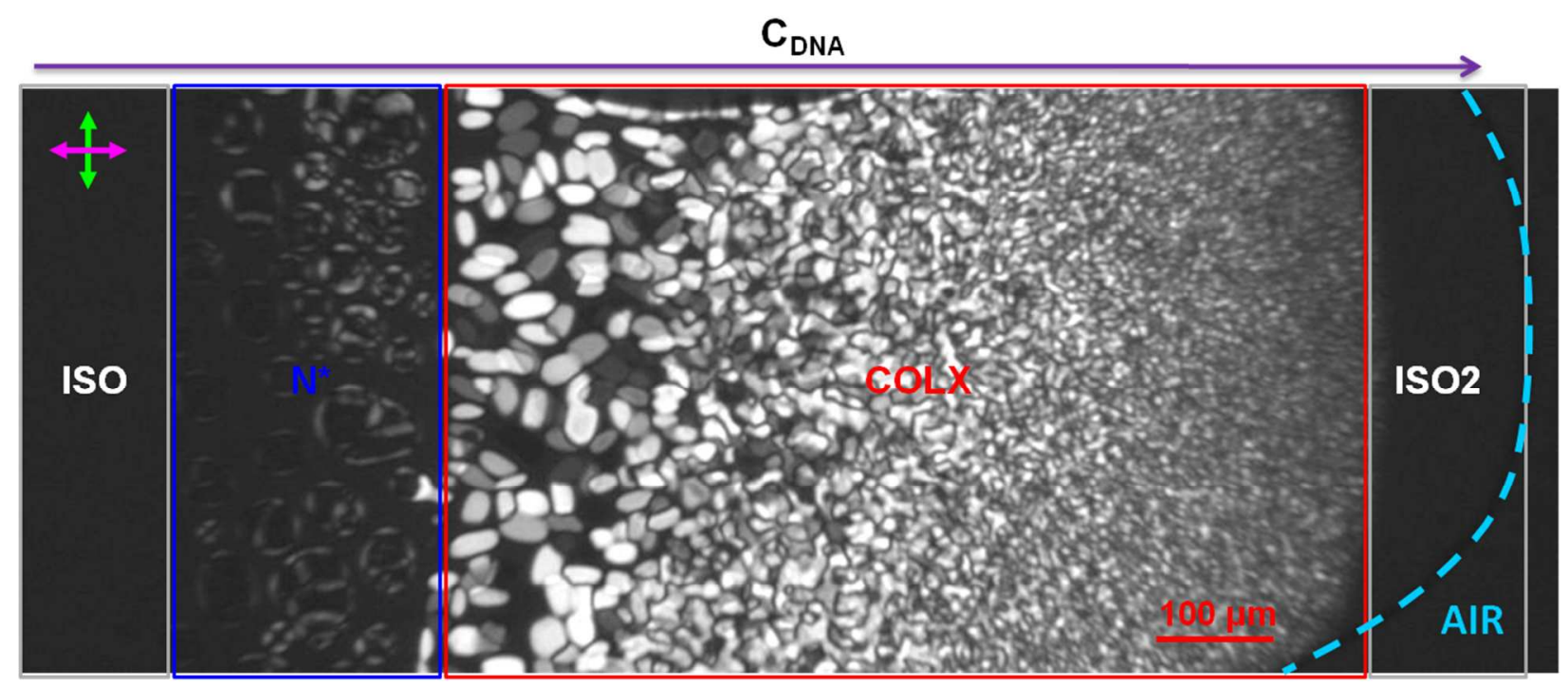

Fig. S7 - B/W PTOM image of a drying GCCG sample in a flat cell.

In Fig. S8 we show the T dependence of coexisting phases. Panes $\mathrm{B}, \mathrm{C}$ and $\mathrm{E}$ show coexistence after equilibration and centrifugation at a given $\mathrm{T}$ (magenta borders). These panes also show that the ISO phase coexisting with LC phases develops LC ordered domains when cooled. Analogously, the LC phases coexisting at a given T with ISO, when heated melt into the ISO state. When a COLX-ISO2 coexistence is gravitationally separated, we find that the ISO2 phase develops COLX ordering when cooled, while the COLX melts into ISO or ISO2 phase when heated. 

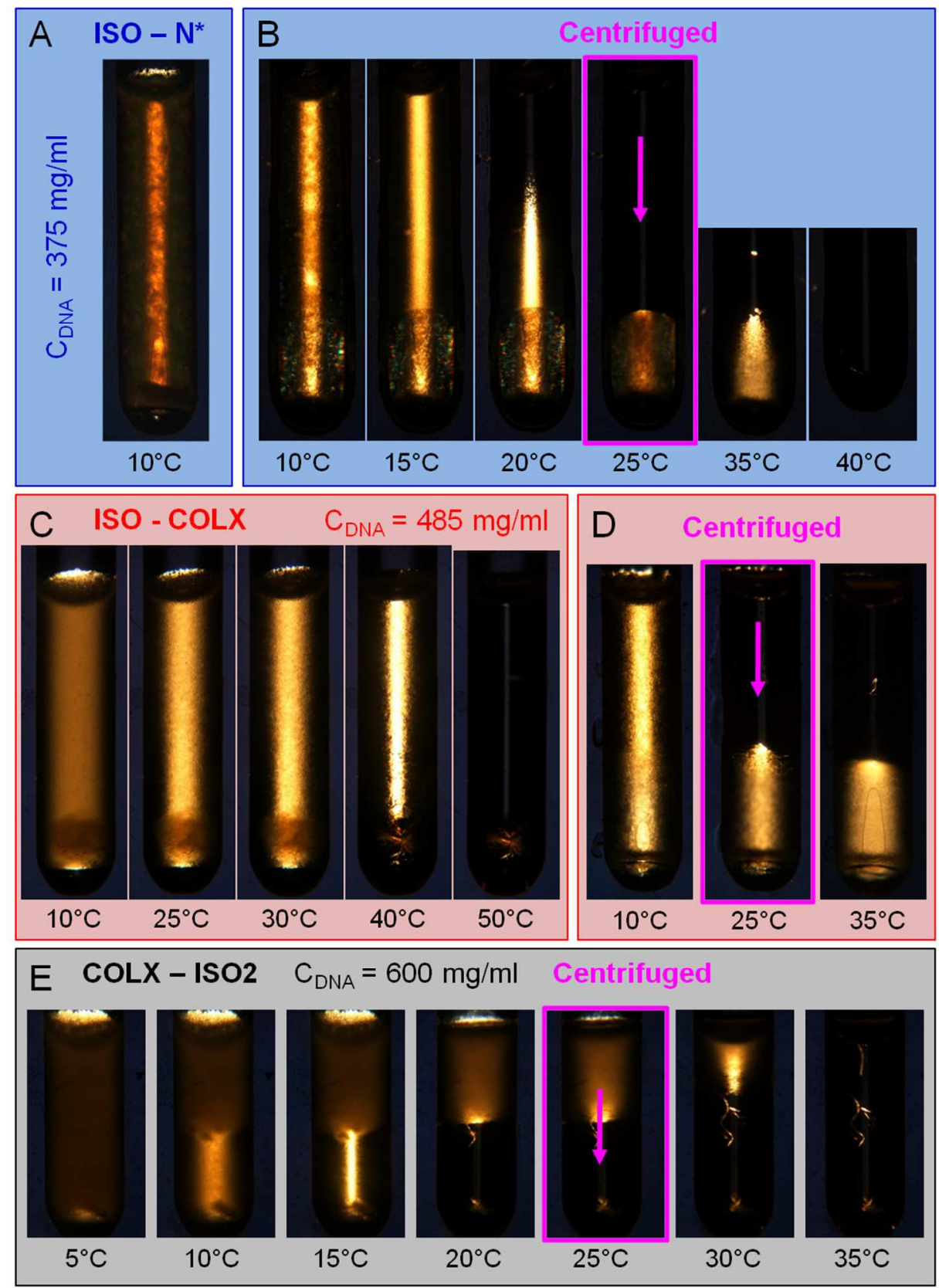

Fig. S8 - Pictures of the $1.5 \mathrm{~mm}$ diameter capillary at different $C_{D N A}$ and $T$. A) ISO - $N^{*}$ coexistence before centrifugation; B) sedimentation of $N^{*}$ phase after centrifugation at $T=25^{\circ} \mathrm{C}$ (magenta border); $C$ ) ISO - COLX coexistence before centrifugation: the COLX phase grows as $T$ is lowered; D) sedimentation of COLX phase centrifugation at $T=25^{\circ} \mathrm{C}$ (magenta border); E) sedimentation of ISO2 phase after centrifugation at $T=25^{\circ} \mathrm{C}$ (magenta border). 


\section{REFERENCES}

(1) De Michele, C.; Bellini, T.; Sciortino, F. Self-Assembly of Bifunctional Patchy Particles with Anisotropic Shape into Polymers Chains: Theory, Simulations, and Experiments. Macromolecules 2012, 45, 10901106.

(2) De Michele, C.; Rovigatti, L.; Bellini, T.; Sciortino, F. Self-Assembly of Short DNA Duplexes: From a Coarse-Grained Model to Experiments through a Theoretical Link. Soft Matter 2012, 8, 8388-8398.

(3) Kuriabova, T.; Betterton, M. D.; Glaser, M. A. Linear Aggregation and Liquid-Crystalline Order: Comparison of Monte Carlo Simulation and Analytic Theory. J. Mater. Chem. 2010, 20, 10366-10383.

(4) Vroege, G. J.; Lekkerkerker, H. N. W. Phase Transitions in Lyotropic Colloidal and Polymer Liquid Crystals. Reports Prog. Phys. 1992, 55, 1241-1309.

(5) SantaLucia, J.; Hicks, D. The Thermodynamics of DNA Structural Motifs. Annu. Rev. Biophys. Biomol. Struct. 2004, 33, 415-440.

(6) Peyret, N. Prediction of Nucleic Acid Hybridization: Parameters and Algorithms, Wayne State University, Detroit, 2000.

(7) Yakovchuk, P.; Protozanova, E.; Frank-Kamenetskii, M. D. Base-Stacking and Base-Pairing Contributions into Thermal Stability of the DNA Double Helix. Nucleic Acids Res. 2006, 34, 564-574.

(8) Mariani, P.; Spinozzi, F.; Federiconi, F.; Amenitsch, H.; Spindler, L.; Drevensek-olenik, I. Small Angle XRay Scattering Analysis of Deoxyguanosine 5 ' -Monophosphate Self-Assembing in Solution : Nucleation and Growth of G-Quadruplexes. J. Phys. Chem. B 2009, 113, 7934-7944.

(9) Horowitz, E. D.; Engelhart, A. E.; Chen, M. C.; Quarles, K. a; Smith, M. W.; Lynn, D. G.; Hud, N. V. Intercalation as a Means to Suppress Cyclization and Promote Polymerization of Base-Pairing Oligonucleotides in a Prebiotic World. Proc. Natl. Acad. Sci. U. S. A. 2010, 107, 5288-5293.

(10) Straley, J. P. Theory of Piezoelectricity in Nematic Liquid Crystals, and of the Cholesteric Ordering. Phys. Rev. A. 1976, 14, 1835-1841.

(11) Frezza, E.; Ferrarini, A.; Kolli, H. B.; Giacometti, A.; Cinacchi, G. Left or Right Cholesterics? A Matter of Helix Handedness and Curliness. Phys. Chem. Chem. Phys. 2014, 16, 16225-16232.

(12) Cherstvy, A. G.; Kornyshev, A. A.; Leikin, S. Torsional Deformation of Double Helix in Interaction and Aggregation of DNA. J. Phys. Chem. B 2004, 108, 6508-6518.

(13) Cherstvy, A. G. DNA Cholesteric Phases: The Role of DNA Molecular Chirality and DNA-DNA Electrostatic Interactions. J. Phys. Chem. B 2008, 112, 12585-12595. 